\title{
EL NACIMIENTO DE UNA NUEVA ESPECIALIZACIÓN
}

\author{
Juan Carlos Galvis Rincón MD*
}

urante años el estudio del ser humano se realizó en estado de reposo. Es así como la fisiología, base de nuestro conocimiento, nos enseña el funcionamiento de esa máquina perfecta que es nuestro cuerpo, pero sin tener en cuenta la razón del mismo que es el movimiento. La mitad de nuestro cuerpo es músculo, el encargado de generar ese tan preciado don que es el desplazamiento voluntario, que permite nuestra independencia y libertad y exige a los demás sistemas su máximo esfuerzo. Quien si no él modifica la velocidad de contracción miocárdica, la hemodinámica, el intercambio gaseoso, la liberación hormonal, la industriosa labor metabólica para el aseguramiento energético y por consiguiente el ordenamiento termodinámico corporal que lleva en últimas a controlar la relación del catabolismo y el anabolismo que gobierna el moldeamiento celular día a día. Por eso el estudio actual del ser humano debe incluir otro tipo de fisiología y es la queocurre durante el movimiento, durante el esfuerzo en condiciones habituales o en condiciones extremas producidas por la temperatura, la humedad, la presión barométrica o la hidrostática, la gravedad, el ejercicio prolongado o extremo o durante la práctica deportiva. El entendimiento de todos los cambios funcionales posibles nos permitirá anticiparnos a eventos patológicos obteniendo así estrategias de prevención útiles en el estado más común del ser humano que es estar despierto y trabajando.

La actividad física que es definida como cualquier contracción muscular que genere un gasto energético, tiene ahora un nuevo centro de desarrollo académico y especial para la comunidad médica en el seno de la Fundación Universitaria de Ciencias de la Salud y su

Especialista en Medicina del Deporte. Hospital Infantil Universitario de San José. Instructor asistente Fundación Universitaria de Ciencias de la Salud, Gerente de la IPS del Deporte UMAF LTDA. Bogotá DC. Colombia.

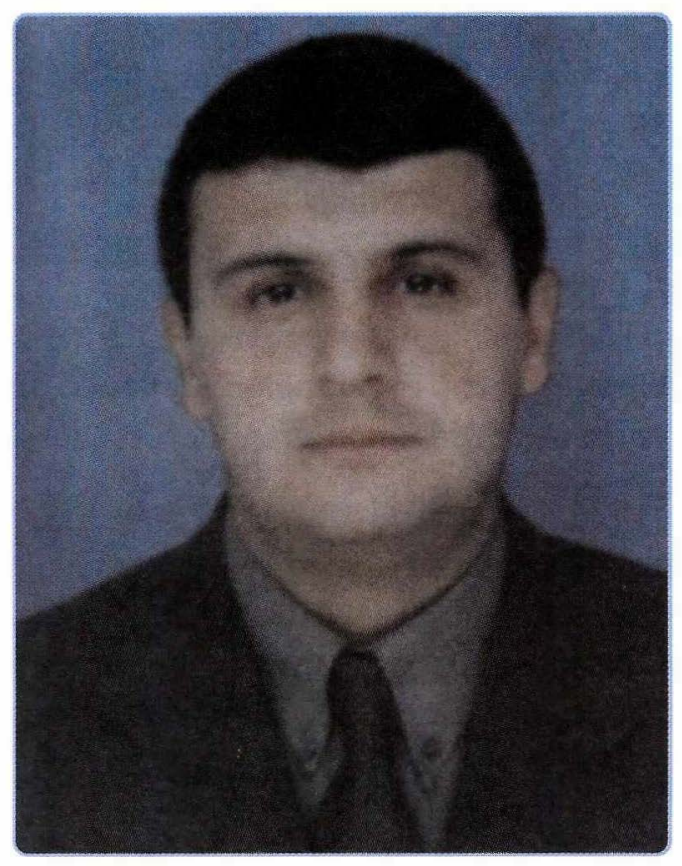

Facultad de Medicina, como una especialización que requirió la estrecha vinculación de la novel rama con las ciencias básicas para diseñar un curso que permite la profundización en las matemáticas para predecir comportamientos, el álgebra y la geometría para optimizar la comunicación gráfica, la trigonometría para el entendimiento de los ciclos, la física para construir modelos y permitir el diseño de los laboratorios de fisiología del esfuerzo y biomecánica, lo cual generará la investigación suficiente para darnos a conocer mediante la publicación científica. La integración necesaria con diferentes especialidades que permiten una sinergia exitosa como ortopedia, cardiología, medicina interna, neumología, endocrinología, pediatría, geriatría, obstetricia, medicina del trabajo entre otras, cuyos resultados son evidentes y conocidos. 
La medicina de la actividad física y del deporte como especialización médica tiene la obligación de prescribir ejercicio en el paciente sano dentro de los programas de promoción de la salud y prevención primaria, lo que amplía el menú de usuarios potenciales a atender, en el paciente con factores de riesgo de cualquier patología para respetar la prevención secundaria y en el enfermo crítico para realizar el proceso de rehabilitación integral. Es así como puede estar presente en cualquier nivel de atención intrahospitalaria y dentro del plan obligatorio de salud. El ejercicio es la base de la rehabilitación cardiovascular, metabólica, pulmonar y osteomusculoarticular. El especialista está en capacidad de construir planes de entrenamiento para el ejercicio sistémico y para el deporte recreativo, aficionado y de alto rendimiento, y adquiere las habilidades necesarias para el manejo de las patologías propias del esfuerzo a nivel osteomusculoarticular y de cualquier sistema implicado, es decir, puede desempeñarse por fuera del sistema de salud colombiano.

Si bien en la actualidad existen tres programas de especialización en Medicina del Deporte en Colombia, ellos centran sus esfuerzos en el estudio y manejo del deportista y buscan sus centros de práctica donde se encuentran sus pacientes, en el sistema Coldeportes.

Esta especialización médica requería por lo tanto un lugar propio de operación por lo que se construyó la Unidad Médica de Actividad Física y Deporte UMFIDE en el corazón deportivo de Bogotá, dentro de las instalaciones del Hospital Infantil Universitario de San José bajo el nombre de la IPS del Deporte, lo que permitió abrir las puertas de nuestro hospital a pacientes sanos y a deportistas. En la IPS del Deporte se realiza toda la rehabilitación del HIUSJ y ocupa cerca del $40 \%$ de toda la consulta externa, con más de 3.000 sesiones de tera- pia física ambulatoria, cerca de 1.000 sesiones mensuales en los pacientes hospitalizados, incluyendo terapia ocupacional y fonoaudiología, más de 100 sesiones de rehabilitación cardíaca y metabólica y al menos la rehabilitación de 20 deportistas cada mes. La consulta en medicina deportiva que por supuesto es particular, asciende a 70 eventos mensuales. Cuenta con un grupo de 300 pacientes dentro de un programa preventivo de enfermedad cardiovascular que asisten todas las semanas para realizar rutinas de ejercicio con resultados maravillosos en cuanto a calidad de vida. La IPS del Deporte asiste a múltiples empresas para la prevención del accidente deportivo dentro de programas incluidos en riesgos profesionales y orienta a deportistas aficionados y de alto rendimiento, teniendo en la actualidad una campeona mundial y récord mundial en patinaje, y un patinador seleccionado al mundial de China 2009 como producción propia.

En el área de investigación el posgrado de medicina de la actividad física y del deporte ha centrado esfuerzos en dos líneas de investigación muy relacionadas con la inactividad física que son el sedentarismo y la sarcopenia. Participa en forma activa en el diseño y creación de programas empresariales para estilos de vida saludable e intrahospitalarios como la rehabilitación del paciente crítico.

Gracias a la visión progresista de la Sociedad de Cirugía de Bogotá y a la Fundación Universitaria de Ciencias de la Salud, contamos en la actualidad con el espacio físico, académico y precursor de la estrategia más económica y efectiva de salud pública como lo es la actividad física y la tarea más publicitada del ser humano como lo es el deporte, que sin duda nos permitirá ser el principal centro de referencia durante los Juegos Panamericanos de Bogotá 2015. 